\title{
Finger Print Image Compression for Extracting Texture Features and Reconstructing
}

\author{
${ }^{1}$ G.Prasanna Lakshmi, ${ }^{2}$ Dr.J.A.Chandulal, ${ }^{3}$ Dr.Y.Gopala Krishna Patro \\ ${ }^{I}$ Swit, University Of Mumbai \\ ${ }^{2}$ Git Visakhapatnam Gitam University \\ ${ }^{3}$ Bvimit University of Mumbai
}

\begin{abstract}
A progressive fingerprint image compression using ridge detection is performed. The image is decomposed into two components. The first component is the primary component, which contains the ridges and bifurcations; the other component is the secondary component, which contains the textures and the features. The decoding process is based on reconstructing a hybrid surface [1] with the means of ridges. The encoding scheme is performed using the tracing algorithm. The proposed method gives a high compression ratio 50:1 which is higher than the fbi specified. The method is also helpful at recognition as the minutia can be driven directly from the compressed image so decompression is not required.
\end{abstract}

Keywords: Bifurcation, reconstruction, fuzzy logic.

\section{Introduction:}

Compression of fingerprints is an essential step in automated fingerprint identification systems due to the increasing number of the fingerprint records in their databases. To achieve higher compression ratios, regular structure of fingerprint images should be utilized by means of model based coding techniques $[2, \mathbf{3}$, and 4].

The fingerprint images can be compressed in two different categories 1) compression is done by extracting the ridges and other texture features. These types of implementations don't have reconstruction of the image. 2) Compression is done using the image transform such as wavelets. These

techniques consume high computational time .The proposed method is capable of both the methods .The image can be reconstructed if required else for authentication the data can be extracted directly from the compressed image .As they are no wavelet/complex transform for compression the computational time as well compression ratio are better than the fbi specified.

The rest of the paper as follows, section 2 contains the previous methods implemented and related work. Section 3 contains the proposed method for extraction of ridges as the primary component and compression, encoding of the fingerprint as the second component. Section 4 contains the reconstruction of the finger print. Section 5 contains the results for the extracted ridges, compression, encoding and an analysis report with the previous methods.

\section{Related works:}

King Saud University, Riyadh 11421 has proposed a feature extraction model in which edge detection and Huffman coding takes a major role[5].Ilker Ersoy, Fikret E T C proposed the method for ridge extraction and the decoding of the fingerprint in [6] .The proposed method used above two references for a better compression as well reconstruction "hand book of finger print recognition" has proposed the various recognition techniques in which the proposed method the compression is performed making the recognition simpler. Fuzzy logic has been implemented for the enhancement in [5] which is implemented for the ridges extraction.

Ridge and valley extraction:

\section{Proposed method}

The ridge and valley can be extracted using binirazation and thinning methods. the end points ,bifurcations are extracted from the image which is similar to edge detection in [5].As the edge detection on finger print doesn't give more accuracy for reconstruction of image so we are replacing the parameters with ridges . 


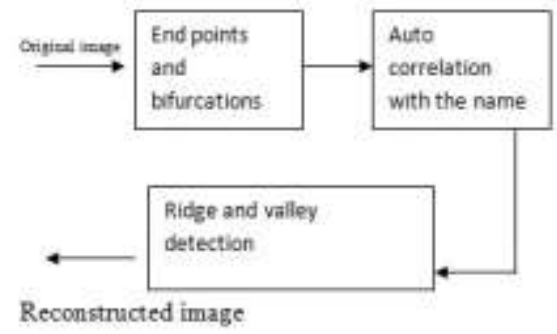

Fig 1: the block diagram of the ridge and valley extraction from the finger print image

\section{Compression:}

The applications benefiting from this algorithm are in transmitting the images over low bandwidth channels such as wireless channels and telephone lines. Examples for such applications are access to remote fingerprint image databases, electronic shopping, and security systems. The last couple of years have seen an explosive growth of business-to-customer activities over the Internet. The total dollar value of these webbased transactions is over several billion US dollars [7]. The present transaction over the web is not capable of assuring that the rightful owner of the credit card is the one who initiate the transaction. Furthermore, the present practice is not capable of linking the transaction to the rightful owner to the credit card. Very soon, credit card owners and credit card issuers will demand more reliable and secure authentication techniques that link the owner of the credit card and the transaction with the help of fingerprint [8]. For these applications, it is important for the viewer to recognize the content as early as possible in the transmission. In this section, encoding of primary and secondary components will be present for the primary component; a combination of arithmetic code and vector quantization (VQ) is designed for the nameplates. This encoding process reduced the required bit to transmit the compressed ridge detected fingerprint images [4].

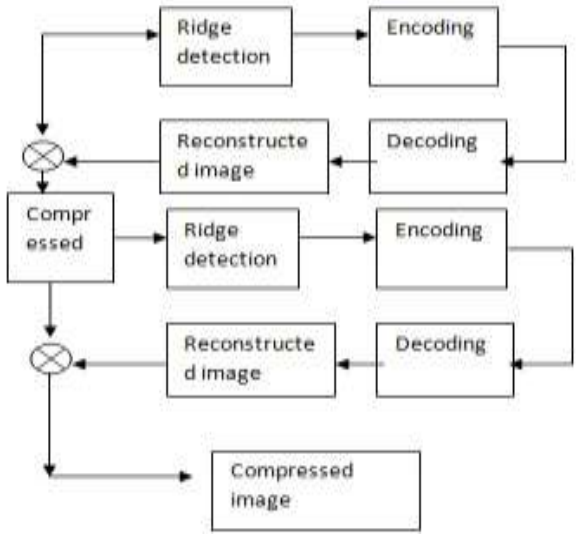

Fig2: compression scheme for the proposed method

As in fig 2 the resultant image is compressed using the encoding algorithms like arithmetic encoding as in [9].The encoding supports the general images for binary images using tracing algorithm similar to it taking 5 pixels per ridge.

\section{Reconstruction of the image:}

Given a sparse data $d(x, y)$ containing gray values on ridges and valleys: the fingerprint image $f(x, y)$ can be obtained by minimizing hybrid energy functional [9].

$$
E_{\lambda t}(f(x, y))=\iint_{\Omega}\left\{\beta(f-d)^{2}+\lambda\left[(1-\tau)\left(f_{x}^{2}+f_{y}^{2}\right)\right]\right.
$$

Where $\mathrm{x}>0$ is the real-valued regularization parameter which controls the compromise between two terms and is the real-valued continuity control parameter. In this functional, the first term on the right hand side is a measure of the closeness of the solution $f(x . y)$ to the data $d(s: y)$, and the second term, which is a convex combination of membrane and plate functional [3], is a measure of the smoothness. By sweeping these two parameters in their specified range, one can generate the AT - space representation of the image. The properties of this space are studied in detail in [6]. 


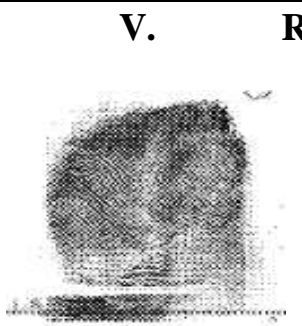

(a)

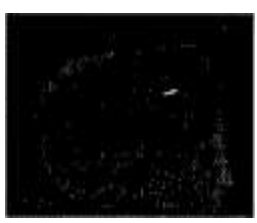

(c)
Results:

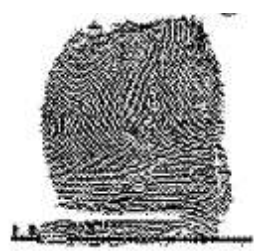

(b)

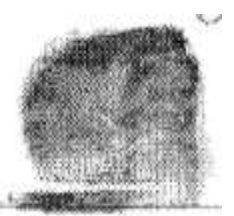

(d)

Fig 3.The results of proposed method

Fig 3(a) is the finger print image given as input for the algorithm with pre processing as stated in [10]. Fig3(b) is the ridges of the finger print input .fig 3(c) is the valleys in the input image .fig 3(d) is the reconstructed image using reconstruction algorithm .

\begin{tabular}{|l|l|l|}
\hline Image & Wsq (fbi) & Proposed method \\
\hline 1 & 12.2 & 9.0 \\
\hline 2 & 15.7 & 11.0 \\
\hline 3 & 18.2 & 14.0 \\
\hline 4 & 18.6 & 14.1 \\
\hline 5 & 15.9 & 10.5 \\
\hline 6 & 16.0 & 9.6 \\
\hline 7 & 11.4 & 6.7 \\
\hline
\end{tabular}

Table 1: comparison of compression ratio of fbi specified method compression and our proposed method.

\section{Conclusion:}

The proposed method can be used for the applications using texture and reconstruction of finger print. The compression method proposed is extracted from the recognition algorithms. So the computation time can be used efficiently by parallel processing.

\section{References:}

[1]. A new pipeline design for binarization and thinning of fingerprint images.

[2]. T. Acar, M. Gokmcn, "Image coding using weak membrane model of images:" Proc. Vis. Comm. and Image Prac., SPIE 01.2308, Sept,. 1994, Chicago, pp. 1221-1230.

[3]. A. Blake, A. Zisserman, Visual Reconstruction”, MIT Press, Cambridge, USA, August 1987.

[4]. J.N. Bradley, C.M. Brislawn, "The wavelet/scalar quantization compression standard for digital fingerprint images," PROC. OF CONF.SIGNAL, IMAGE PWC AND UPPL.ANNECY, FRANCE, JUNE 1996, PP.245-247.

[5]. "PROGRESSIVE FINGERPRINT IMAGES COMPRESSION USING EDGE DETECTION TECHNIQUE".

[6]. A MODEL-BASED APPROACH FOR COMPRESSION OF FINGERPRINT IMAGES.

[7]. N. K. Ratha, J. H. Conne, and R. M. Bolle, "Secure data hiding in wavelet compressed fingerprint images", Proc. of Int'l Conf. on ACM Multimedia 2000 workshops, pp. 127-130, Los Angeles, California, 2000.

[8]. J.K. Yan and D. J. Sakrison, .Encoding of images based on a two component source model., IEEE Trans. Commun., Vol. 25, PP.1315-1322, Nov. 1997

[9]. Arithmetic Coding For Data Coiupression Ian H.Willen,Radford M.Neal and John G.Cleary.

[10]. Fingerprint Analysis and Matching using Fuzzy logic Design .IOSRJCE (July-Aug), pp 04-08, ISSN: 2278-0661 volume 1, Issue 6(IOSR JCE). 